\title{
Blendas de Poli (Ácido Lático-co-ácido Glicólico)/ Poli (Ácido Lático): Degradação in vitro
}

\author{
Camila A. de Rezende \\ Faculdade de Engenharia Mecânica, UNICAMP \\ Eliana Ap. R. Duek \\ Centro de Ciências Médicas e Biológicas, PUC-SP
}

\begin{abstract}
Resumo: Placas de copolímero de poli(ácido lactico-co-glicólico) têm sido produzidas e usadas como implantes que degradam e são absorvidos pelo organismo. Implantes que podem ser absorvidos apresentam vantagens em relação aos implantes metálicos. Nesse trabalho, foram obtidas placas a partir de blendas de poli(ácido lactico-co-glicólico)/ poli(ácido lático), (PLGA/PLLA) e caracterizadas durante o processo de degradação in vitro. Verificou-se que as blendas são imiscíveis e a estabilidade térmica das mesmas aumenta com a proporção de PLLA. O grau de cristalinidade também aumenta com a proporção de PLLA na amostra e com o tempo de degradação. Além disso, verificou-se que o PLGA degrada rapidamente e sua presença e quantidade modifica nitidamente a morfologia das blendas.
\end{abstract}

Palavras-chave: Blendas, poli(L-ácido lático), poli(L-ácido lático-co-ácido glicólico), degradação.

\section{Poly (Lactide-co-Glycolide) Acid/Poly (Lactic Acid) Blends: In vitro Degradation}

Abstract: Plates of the copolymer poly (lactide-co-glycolide) acid have been produced and used as implants that degrade and are absorbed by the organism. Implants that can be absorbed are advantageous in comparison with metallic implants. In this work, plates of the blend poly (lactide- co- glycolide) acid/ poly lactic acid (PLGA/ PLLA) were made and characterized during their in vitro degradation process. It was found that blends are immiscible and that their thermal stability increases with the proportion of PLLA in the blend. The crystallinity degree also increases with the proportion of PLLA in the sample and with degradation time. Besides, it was verified that PLGA is degraded quickly and its presence and amount modifies clearly the morphology of the blends.

Keywords: Blends, poli(L-lactic acid), poli(L-latic acid-co-glycolic acid), degradation.

\section{Introdução}

Os biomateriais poliméricos e seus compósitos podem ser classificados como bioestáveis, parcialmente biodegradáveis ou totalmente biodegradáveis. Os polímeros bioestáveis são praticamente inertes, causam uma resposta mínima por parte do tecido e mantêm suas propriedades durante anos. Os implantes totalmente absorvíveis são hidroliticamente instáveis e possuem caraterísticas de biodegradação tais que os tornam capazes de serem totalmente elimina- dos pelo metabolismo corpóreo até certo tempo após o implante. Os polímeros parcialmente absorvíveis também são hidroliticamente instáveis, mas como não conseguem ser totalmente metabolizados pelo organismo e eliminados deste, não dispensam a necessidade da segunda cirurgia que retira o implante após a cicatrização do tecido ${ }^{[1]}$.

Até 1960, polímeros hidroliticamente instáveis eram considerados uma descoberta desastrosa, entretanto, com o avanço das pesquisas, esse materiais passaram a despertar o interesse da medicina especial-

Autor para correspondência: Eliana A. R. Duek, Centro de Ciências Médicas e Biológicas, PUC, Praça Dr. José Ermírio de Moraes, 290, CEP: 18030-230 Sorocaba, SP. E-mail: eliduek@fem.unicamp.br 
mente devido à possibilidade de serem utilizados em implantes temporários ${ }^{[2]}$.

Em muitas aplicações clínicas, não há a necessidade de um implante permanente e, é graças a este vasto campo de aplicações temporárias, que os polímeros bioabsorvíveis são tão requisitados ${ }^{[1]}$.

Uma propriedade interessante nos materiais absorvíveis é que eles vão transferindo tensão gradualmente para o osso em cicatrização à medida que degradam; isto evita um problema muito comum nos implantes metálicos que é a sobrecarga de tensão que ocorre no órgão fraturado quando o material implantado é feito de um outro mais rígido que ele próprio. Uma outra vantagem é a eliminação de uma segunda cirurgia necessária para retirar o implante metálico assim que o local da fratura está reconstituído, o que é financeiramente e psicologicamente benéfico para o paciente ${ }^{[3]}$.

Além disto, um material polimérico não causa processos inflamatórios decorrentes da liberação de íons metalicos ao redor do implante, ao contrário, libera substâncias que podem até gerar energia ou participar de processos de metabolização proteica no organismo. É preciso levar em conta que implantes temporários também podem causar processos inflamatórios devido a outros fatores, mas estes têm sido cada vez mais compreendidos e minimizados. Assim, os materiais absorvíveis fazem-se cada vez mais indicados para fixação de fraturas internas ${ }^{[1]}$.

Para aplicações em ortopedia deve-se avaliar uma série de fatores. Primeiro o dispositivo deve ter o design próprio e a resistência adequada. Deve ser manufaturado sobre controle, de forma a não incorporar defeitos no implante. A seleção do material é importante e deve garantir biocompatibilidade e resistência a corrosão durante o tempo necessário para recuperação do osso ${ }^{[3]}$.

\section{Experimental}

As blendas de poli(ácido lático)-co-(ácido glicólico)/poli (ácido lático) [PLGA/PLLA] foram preparadas nas composições 100:0, 70:30, 50:50, 30:70, 0:100 (o primeiro valor na proporção referese ao PLGA e o segundo ao PLLA), através de um processo de fusão, utilizando-se uma mini-injetora Mini Max Molden modelo LMM- 2017.

Quantidades certas de polímeros foram pesadas de acordo com o tamanho do molde e a proporção de

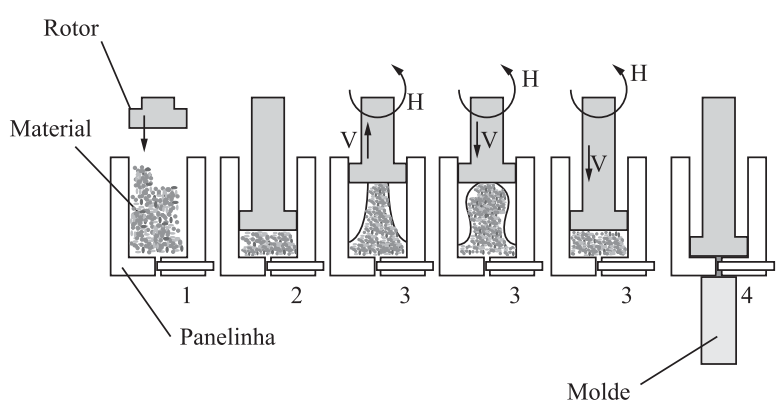

Figura 1. Representação esquemática da preparação das placas.

cada um na blenda. Na Figura 1, há uma representação do processo de preparação das placas.

O material foi colocado na panelinha da extrusora previamente aquecida a uma temperatura de $190^{\circ} \mathrm{C}$ (no caso da extrusão do PLGA puro) ou de $205^{\circ} \mathrm{C}$ (para as demais blendas), conforme esquematizado na etapa 1 da Figura 1. Os polímeros colocados na panelinha foram aquecidos por 1 minuto sem cisalhamento (etapa 2) e depois por 1,5 minuto com cisalhamento horizontal $(\mathrm{H})$ a uma velocidade constante de $5 \mathrm{rpm}$ e cisalhamento vertical (V), conforme a etapa 3 . O material fundido foi injetado num molde, cuja temperatura foi ajustada conforme a composição da blenda, assim como a temperatura usada para fundir o material a ser injetado (etapa 4).

A mistura injetada no molde foi resfriada à temperatura ambiente e, depois disto, a placa foi retirada do molde e armazenada no dessecador.

O processo de degradação das placas foi feito em tampão fosfato $\mathrm{pH} 7,0$ a $37^{\circ} \mathrm{C}$, em tempos de 30 , $60,90,120$ e 150 dias em frascos de vidro tampados. Antes de se submeterem às análises, as placas permaneceram por oito horas em uma estufa a vácuo com temperatura de $50{ }^{\circ} \mathrm{C}$. Após a preparação e a degradação, as placas foram caracterizadas através das técnicas citadas a seguir:

\section{Calorimetria Diferencial de Varredura (DSC)}

Para a realização dos ensaios de DSC, foi utilizado o equipamento da TA Instruments modelo MDSC 2910. As amostras, pesando cerca de $17 \mathrm{mg}$, foram levadas até a temperatura de $200{ }^{\circ} \mathrm{C}$ e mantidas nesta temperatura por 5 min até completa fusão, apagando a história térmica da amostra. Em seguida, foram resfriadas até $-20{ }^{\circ} \mathrm{C} \mathrm{e}$, novamente, mantidas a esta temperatura por $5 \mathrm{~min}$; finalmente foram reaquecidas até $200{ }^{\circ} \mathrm{C}$. Tanto o aquecimento quanto o resfriamento foram realizados com uma rampa de aquecimento de $10{ }^{\circ} \mathrm{C} / \mathrm{min}$ e em atmosfera de hélio. 


\section{Análise termogravimétrica (TGA)}

Para a análise de TGA, foi utilizado o equipamento STA 409C da NETZSCH - Gerätebau GmbH Thermal Analysis. As amostras das placas, pesando ao redor de $17 \mathrm{mg}$ foram aquecidas de 25 a $450{ }^{\circ} \mathrm{C}$ a $10{ }^{\circ} \mathrm{C} / \mathrm{min}$, sob atmosfera de hélio.

\section{Análise dinâmico-mecânica (DMA)}

A análise dinâmico-mecânica foi realizada no equipamento DMA - Dynamic Mechanical Analysis - 242 da NETZSCH, sob ar. As amostras foram resfriadas até $-100{ }^{\circ} \mathrm{C}$ e, em seguida, aquecidas até $200{ }^{\circ} \mathrm{C}$, com rampa de aquecimento de $5^{\circ} \mathrm{C} / \mathrm{min}$. A freqüência utilizada foi de $1 \mathrm{~Hz}$, a amplitude de $15 \mu \mathrm{m}$ e a força dinâmica de $0,5 \mathrm{~N}$. Utilizou-se o modo tipo tração no sistema Tension. Para melhor ajuste das condições impostas as amostras foram submetidas a uma temperatura de $-40{ }^{\circ} \mathrm{C}$. O ensaio dinâmico-mecânico através do módulo de tração ou flexão (DMA) só foi realizado para as placas não degradadas.

\section{Microscopia eletrônica de varredura (MEV)}

Amostras de fratura das placas, feitas em $\mathrm{N}_{2}$ líquido, foram colados em um suporte metálico e recobertos com ouro, utilizando-se um metalizador de amostras Sputer Coater BAL-TEC SCD 050. Em seguida, as amostras foram observadas no microscópio eletrônico de varredura JEOL JXA 840A, utilizando tensão de 10 kV.

\section{Difratometria de raios- $X$}

As medidas de difração de raios-X das placas foram realizadas em um equipamento "XD-3A Shimadzu X-ray diffractometer" utilizando-se radiação de $\mathrm{CuK} \alpha$ e faixa angular entre 5 e $50^{\circ}$, a $30 \mathrm{kV}$ de tensão e $20 \mathrm{~mA}$.

\section{Resultados e Discussão}

Os resultados obtidos a partir da análise termogravimétrica (TGA) para as amostras das placas de PLGA/ PLLA mostraram que elas apresentam apenas um estágio de perda de massa em todos os tempos de degradação, como pode ser verificado na Figura 2 para a blenda $70: 30$ e na Figura 3 para as blendas 0:100, 70:30, 50:50, 30:70 em 60 dias de degradação.

Na Tabela1, encontram-se os valores de Td obtidos para todas as blendas em diferentes estágios de degradação. Pode-se verificar que o valor em que a

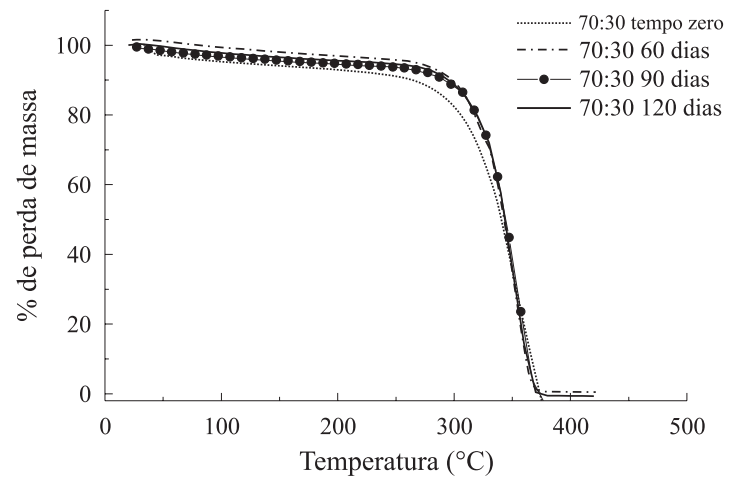

Figura 2. Curvas obtidas a partir de TGA para as blendas de PLGA/ PLLA 70:30 em diferentes tempos de degradação.

perda de massa é máxima, indicado por Td, aumentou com o aumento da proporção de PLLA nas blendas e que, portanto, a estabilidade térmica das mesmas também aumentou com a proporção deste polímero. Nas placas com PLLA puro praticamente não se observa degradação durante o período de tempo estudado (6 meses). Por outro lado, a degradação do PLGA foi bastante efetiva, tanto que, após 30 dias de degradação não foi mais possível realizar análises.

Para a blenda PLGA/PLLA 50:50, verificou-se que há um aumento inicial na sua estabilidade térmica entre os tempos 0 e 30 dias de degradação, depois

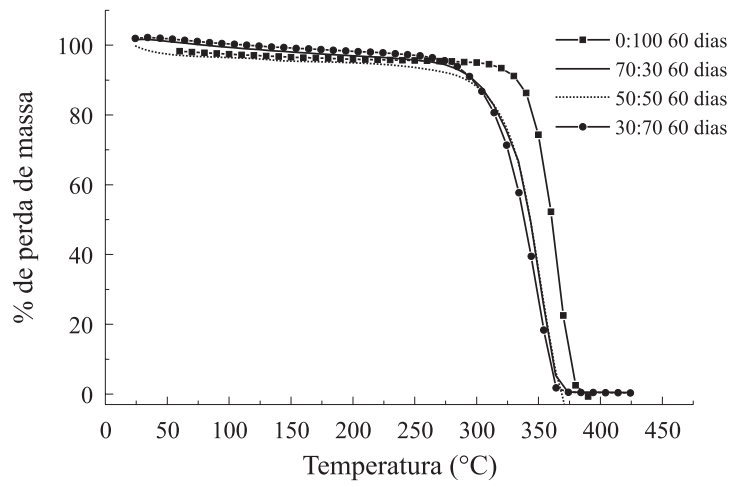

Figura 3. Curvas obtidas a partir de TGA para as blendas de PLGA/ PLLA 0:100, 70:30, 50:50 e 30:70 em 60 dias de degradação.

Tabela 1. Valores de temperatura em que a perda de massa é máxima $\left(\mathrm{Td} /{ }^{\circ} \mathrm{C}\right)$ obtidos a partir das análises de TGA para as blendas de (PLGA/ PLLA) em diferentes tempos de degradação.

\begin{tabular}{cccccc}
\hline $\begin{array}{c}\text { Tipos de } \\
\text { blendas }\end{array}$ & $\mathbf{t}_{\text {zero }}$ & $\mathbf{t}_{\mathbf{3 0 ~ d i a s}}$ & $\mathbf{t}_{\mathbf{6 0} \text { dias }}$ & $\mathbf{t}_{\mathbf{9 0 \text { dias }}}$ & $\mathbf{t}_{\mathbf{1 2 0 \text { dias }}}$ \\
\hline $100: 0$ & 357 & 312 & & & \\
$70: 30$ & 362 & 349 & 356 & 356 & 346 \\
$50: 50$ & 342 & 362 & 352 & 348 & 352 \\
$30: 70$ & 359 & 362 & 352 & 352 & 347 \\
$0: 100$ & 365 & 369 & 368 & 347 & 361 \\
\hline
\end{tabular}


disso, a Td diminuiu lentamente até 120 dias. Provavelmente, o aumento inicial deva-se à perda de PLGA; com a degradação do copolímero, restou apenas PLLA ou um grande excesso deste na amostra e, então, a estabilidade térmica da blenda aumenta, porque o PLLA tem estabilidade maior. Depois de todo o copolímero ter sido eliminado, a estabilidade começa a cair lentamente, devido ao processo de degradação do PLLA, que é bastante lento.

A análise de calorimetria diferencial de varredura (DSC) forneceu valores de temperatura de transição vítrea (Tg) para as várias composições de blendas submetidas a vários tempos de degradação que se encontram na Tabela 2.

Nas análises realizadas antes da degradação, observou-se a o aparecimento de duas temperaturas de transição vítrea distintas, referentes a cada um dos polímeros constituintes da blenda. Após 30 dias de degradação, existe apenas um valor de $\mathrm{Tg}$, referente ao PLLA, provavelmente porque o PLGA degradouse completamente, o que pode ser confirmado pelo fato não ter sido possível realizar nenhum tipo de análise com as placas de copolímero puro após 30 dias de degradação. Observando-se os tempos 0 e 30 dias para as blendas que continham PLGA, verificou-se uma diminuição acentuada nos valores de Tg. Essa diminuição deve-se à degradação do PLGA. Para os outros tempos de degradação verificou-se uma variação muito pequena na $\mathrm{Tg}$ para qualquer uma das blendas, pois a degradação do PLLA é lenta.

Na Tabela 3, encontram-se os valores de tempe-

Tabela 2. Valores de temperatura de transição vítrea $\left(\mathrm{Tg} /{ }^{\circ} \mathrm{C}\right)$ obtidos a partir das análises de DSC para as blendas de (PLGA/PLLA) em diferentes tempos de degradação

\begin{tabular}{ccccccc}
\hline \multicolumn{2}{c}{$\begin{array}{c}\text { Tipos de } \\
\text { blendas }\end{array}$} & $\mathbf{t}_{\text {zero }}$ & $\mathbf{t}_{30 \text { dias }}$ & $\mathbf{t}_{60 \text { dias }}$ & $\mathbf{t}_{\mathbf{9 0} \text { dias }}$ & $\mathbf{t}_{\mathbf{1 2 0} \text { dias }}$ \\
\hline \multirow{2}{*}{$100: 0$} & $1^{\mathrm{o}} \mathrm{aq}$ & $33 ; \mathrm{xx}$ & 38 & - & - & - \\
& $2^{\mathrm{o}} \mathrm{aq}$ & $44 ; \mathrm{xx}$ & 4 & - & - & - \\
\hline \multirow{2}{*}{$70: 30$} & $1^{\mathrm{o}} \mathrm{aq}$ & $47 ; 61$ & 59 & 58 & 57 & 56 \\
& $2^{\mathrm{o}} \mathrm{aq}$ & $45 ; 58$ & 53 & 56 & 56 & 56 \\
\hline \multirow{2}{*}{$50: 50$} & $1^{\mathrm{o}} \mathrm{aq}$ & $43 ; 60$ & 58 & 64 & 62 & 57 \\
& $2^{\mathrm{o}} \mathrm{aq}$ & $45 ; 61$ & 53 & 58 & 58 & 57 \\
\hline \multirow{2}{*}{$30: 70$} & $1^{\mathrm{o}} \mathrm{aq}$ & $52 ; 63$ & 60 & 64 & 63 & 58 \\
& $2^{\mathrm{o}} \mathrm{aq}$ & $45 ; 61$ & 55 & 58 & 58 & 53 \\
\hline \multirow{2}{*}{$0: 100$} & $1^{\mathrm{o}} \mathrm{aq}$ & $\mathrm{xx} ; 72$ & 64 & 67 & 65 & 65 \\
& $2^{\mathrm{o}} \mathrm{aq}$ & $\mathrm{xx} ; 63$ & 60 & 62 & 62 & 61 \\
\hline
\end{tabular}

Tabela 3. Valores de temperatura de cristalização $\left(\mathrm{Tc} /{ }^{\circ} \mathrm{C}\right)$ obtidos a partir das análises de DSC para as blendas de (PLGA/PLLA) em diferentes tempos de degradação

\begin{tabular}{|c|c|c|c|c|c|c|}
\hline \multicolumn{2}{|c|}{$\begin{array}{l}\text { Tipos de } \\
\text { blendas }\end{array}$} & $\mathbf{t}_{\text {zero }}$ & $\mathbf{t}_{30 \text { dias }}$ & $t_{60 \text { dias }}$ & $\mathbf{t}_{90 \text { dias }}$ & $t_{120 \text { dias }}$ \\
\hline \multirow{2}{*}{ 100:0 } & $1^{\circ} \mathrm{aq}$ & - & - & - & - & - \\
\hline & $2^{\circ} \mathrm{aq}$ & - & - & - & - & - \\
\hline \multirow{2}{*}{$70: 30$} & $1^{\circ} \mathrm{aq}$ & 105 & 100 & 90 & 88 & 87 \\
\hline & $2^{\circ} \mathrm{aq}$ & 106 & 97 & 96 & 99 & 97 \\
\hline \multirow{2}{*}{$50: 50$} & $1^{\circ} \mathrm{aq}$ & 104 & 90 & 91 & 88 & 100 \\
\hline & $2^{\circ} \mathrm{aq}$ & 106 & 98 & 101 & 101 & 88 \\
\hline \multirow{2}{*}{$30: 70$} & $1^{\circ} \mathrm{aq}$ & 108 & 87 & 89 & 88 & 82 \\
\hline & $2^{\circ} \mathrm{aq}$ & 109 & 97 & 98 & 100 & 93 \\
\hline \multirow{2}{*}{$0: 100$} & $1^{\circ} \mathrm{aq}$ & - & 90 & 105 & 98 & 89 \\
\hline & $2^{\circ} \mathrm{aq}$ & 131 & 106 & 104 & 106 & 103 \\
\hline
\end{tabular}

ratura de cristalização (Tc) para as blendas PLGA/ PLLA submetida a diferentes tempos de degradação. Pôde-se verificar que a Tc obtida para cada uma das blendas tendeu a diminuir à medida que o tempo de degradação aumentou. Na literatura, essa diminuição tem sido atribuída ao efeito de relaxação das cadeias do polímero; de uma maneira geral, cadeias poliméricas de menor massa molar requerem uma menor energia de cristalização ${ }^{[5]}$.

Pode-se então concluir que, a diminuição de Tc deve-se à degradação do material, que gera cadeias de menor massa molar e exigem uma menor energia de cristalização para a formação de novos cristais. Da mesma forma, pode-se explicar porque a entalpia de cristalização $(\Delta \mathrm{Hc})$ tendeu a diminuir à medida que os tempos de degradação aumentaram. Os valo-

Tabela 4. Valores de entalpia de cristalização $\left(\Delta \mathrm{Hc} / \mathrm{J} \mathrm{g}^{-1}\right)$ obtidos a partir das análises de DSC para as blendas de (PLGA/PLLA) em diferentes tempos de degradação

\begin{tabular}{ccccccc}
\hline \multicolumn{2}{c}{$\begin{array}{c}\text { Tipos de } \\
\text { blendas }\end{array}$} & $\mathbf{t}_{\text {zero }}$ & $\mathbf{t}_{\text {30 dias }}$ & $\mathbf{t}_{60 \text { dias }}$ & $\mathbf{t}_{\mathbf{9 0} \text { dias }}$ & $\mathbf{t}_{\mathbf{1 2 0} \text { dias }}$ \\
\hline $70: 30$ & $1^{\circ} \mathrm{aq}$ & - & - & - & - & - \\
& $2^{\circ} \mathrm{aq}$ & 10 & 17 & 5 & 6 & 5 \\
\hline \multirow{2}{*}{$50: 50$} & $1^{\circ} \mathrm{aq}$ & 31 & 1 & 19 & 15 & 18 \\
& $2^{\circ} \mathrm{aq}$ & 41 & 27 & 23 & 24 & 17 \\
\hline $30: 70$ & $1^{\circ} \mathrm{aq}$ & 17 & 10 & 9 & 13 & 3 \\
\hline \multirow{2}{*}{$0: 100$} & $2^{\circ} \mathrm{aq}$ & 41 & 10 & 15 & 21 & 5 \\
\hline & $2^{\circ} \mathrm{aq}$ & - & 10 & 4 & 16 & 2 \\
\hline
\end{tabular}


res de $\Delta \mathrm{Hc}$ obtidos para as blendas a partir das análi ses de DSC encontram-se na Tabela 4.

Os valores da temperatura de fusão (Tm) encontram-se na Tabela 5. Pode-se observar que eles praticamente não variam em função do tempo de degradação ou da composição da blenda, o que indica que a blenda é imiscível. Esse fenômeno já foi observado para as blendas de poli (etileno glicol)/ poli (ácido lático) e para as de poli (3- hidroxibutirato)-co-(3- hidroxivalerato)/ poli (L- ácido lático), pelos autores Younes e colaboradores e Iannace e colaboradores, respectivamente ${ }^{[6,7]}$. Os valores de Tm não variam em função do tempo de degradação porque a fusão é um fenômeno referente apenas ao PLLA.

Os valores do grau de cristalinidade foram calculados a partir dos valores de $\Delta \mathrm{Hc}$ e $\Delta \mathrm{Hm}$ obtidos a partir das análises de DSC. O cálculo é feito utilizando-se a Equação 1 abaixo $^{[8]}$.

$$
\chi=\frac{(\Delta \mathrm{Hm}-\Delta \mathrm{Hc}) \times 100}{\Delta \mathrm{Hm}^{0}}
$$

onde: $\Delta \mathrm{Hm}=$ entalpia de fusão $\left[\mathrm{J}^{-\mathrm{g}^{-1}}\right]$ $\Delta \mathrm{Hc}=$ entalpia de cristalização [J.g $\left.{ }^{-1}\right]$ $\Delta \mathrm{Hm}^{\circ}=93,7 \mathrm{~J} / \mathrm{g}$

O grau de cristalinidade das blendas tendeu a aumentar em função do tempo de degradação. Esse fato é freqüentemente discutido na literatura. Os principais autores sugerem que isso ocorre graças ao rearranjo das cadeias menores que surgem durante $o$ processo de degradação. Essas cadeias se rearranjam e contribuem para o aumento no grau de cristalinidade do polímero, assim como a degradação da parte amorfa também pode contribuir. Alguns autores afirmam que

Tabela 5. Valores de temperatura de fusão $\left(\mathrm{Tm} /{ }^{\circ} \mathrm{C}\right)$ obtidos a partir das análises de DSC para as blendas de (PLGA/PLLA) em diferentes tempos de degradação

\begin{tabular}{ccccccc}
\hline \multicolumn{2}{c}{$\begin{array}{c}\text { Tipos de } \\
\text { blendas }\end{array}$} & $\mathbf{t}_{\text {zero }}$ & $\mathbf{t}_{\mathbf{3 0} \text { dias }}$ & $\mathbf{t}_{\mathbf{6 0} \text { dias }}$ & $\mathbf{t}_{\mathbf{9 0} \text { dias }}$ & $\mathbf{t}_{\mathbf{1 2 0} \text { dias }}$ \\
\hline \multirow{2}{*}{$70: 30$} & $1^{\mathrm{o}} \mathrm{aq}$ & 178 & 175 & 176 & 176 & 177 \\
& $2^{\mathrm{o}} \mathrm{aq}$ & 178 & 172 & 174 & 174 & 173 \\
\hline \multirow{2}{*}{$50: 50$} & $1^{\mathrm{o}} \mathrm{aq}$ & 179 & 177 & 180 & 180 & 174 \\
& $2^{\mathrm{o}} \mathrm{aq}$ & 178 & 172 & 176 & 175 & 178 \\
\hline \multirow{2}{*}{$30: 70$} & $1^{\mathrm{o}} \mathrm{aq}$ & 180 & 180 & 180 & 179 & 178 \\
& $2^{\mathrm{o}} \mathrm{aq}$ & 180 & 175 & 175 & 174 & 171 \\
\hline \multirow{2}{*}{$0: 100$} & $1^{\mathrm{o}} \mathrm{aq}$ & 180 & 180 & 180 & 180 & 180 \\
& $2^{\mathrm{o}} \mathrm{aq}$ & 180 & 179 & 180 & 179 & 180 \\
\hline
\end{tabular}

Tabela 6. Valores de entalpia de fusão $\left(\Delta \mathrm{Hm} / \mathrm{J} \cdot \mathrm{g}^{-1}\right)$ obtidos a partir das análises de DSC para as blendas de (PLGA/PLLA) em diferentes tempos de degradação

\begin{tabular}{ccccccc}
\hline \multicolumn{2}{c}{$\begin{array}{c}\text { Tipos de } \\
\text { blendas }\end{array}$} & $\mathbf{t}_{\text {zero }}$ & $\mathbf{t}_{\mathbf{3 0} \text { dias }}$ & $\mathbf{t}_{\mathbf{6 0} \text { dias }}$ & $\mathbf{t}_{\mathbf{9 0} \text { dias }}$ & $\mathbf{t}_{\mathbf{1 2 0} \text { dias }}$ \\
\hline $70: 30$ & $1^{\mathrm{o}} \mathrm{aq}$ & 29 & 51 & 54 & 64 & 60 \\
& $2^{\circ} \mathrm{aq}$ & 27 & 24 & 58 & 56 & 60 \\
\hline $50: 50$ & $1^{\circ} \mathrm{aq}$ & 73 & 64 & 62 & 63 & 56 \\
\hline $30: 70$ & $2^{\circ} \mathrm{aq}$ & 70 & 59 & 89 & 63 & 67 \\
\hline $0: 100$ & $2^{\circ} \mathrm{aq}$ & 63 & 60 & 60 & 60 & 59 \\
& $2^{\circ} \mathrm{aq}$ & 73 & 61 & 73 & 64 & 54 \\
\hline
\end{tabular}

analisar um processo de degradação não é simplesmente avaliar a relação entre as partes amorfas e cristalinas. A degradação pode ocorrer de uma forma heterogênea, por exemplo, de uma forma mais rápida no centro do dispositivo, onde há um acúmulo de produtos ácidos provenientes da própria degradação. Além disso, o processo pode variar de acordo com a estrutura e a configuração das cadeias poliméricas, bem como com a morfologia do material ${ }^{[9,10]}$.

A partir da análise de DMA, foram obtidos os valores de Tg e de Tm que se encontram na Tabela 8 para as blendas de PLGA/PLLA antes da degradação. Os valores foram obtidos a partir dos parâmetros módulo de perda (E"), que é proporcional à energia dissipada e do fator de perda $(\tan \delta)$, que é a medida da energia dissipada comparativamente com a energia armazenada pelo material.

Pode-se observar que a Tg obtida a partir de E" para o PLLA está em torno de $80^{\circ} \mathrm{C}$ enquanto a do

Tabela 7. Valores de grau de cristalinidade (\%) calculados a partir dos valores de $\Delta \mathrm{Hc}$ e de $\Delta \mathrm{Hm}$ para as blendas de (PLGA/PLLA) em diferentes tempos de degradação

\begin{tabular}{ccccccc}
\hline \multicolumn{2}{c}{$\begin{array}{c}\text { Tipos de } \\
\text { blendas }\end{array}$} & $\mathbf{t}_{\text {zero }}$ & $\mathbf{t}_{\mathbf{3 0} \text { dias }}$ & $\mathbf{t}_{\mathbf{6 0 \text { dias }}}$ & $\mathbf{t}_{\mathbf{9 0} \text { dias }}$ & $\mathbf{t}_{\mathbf{1 2 0} \text { dias }}$ \\
\hline $70: 30$ & $1^{\circ} \mathrm{aq}$ & 25 & 49 & 39 & 47 & 40 \\
& $2^{\circ} \mathrm{aq}$ & 18 & 50 & 56 & 53 & 58 \\
\hline $50: 50$ & $1^{\circ} \mathrm{aq}$ & 45 & 67 & 52 & 51 & 42 \\
\hline $30: 70$ & $2^{\circ} \mathrm{aq}$ & 31 & 34 & 70 & 42 & 53 \\
\hline $0: 100$ & $2^{\circ} \mathrm{aq}$ & 49 & 53 & 54 & 50 & 60 \\
\hline & $2^{\circ} \mathrm{aq}$ & 34 & 54 & 62 & 46 & 52 \\
\hline
\end{tabular}


Tabela 8. Valores de temperatura de transição vítrea $\left(\mathrm{Tg} /{ }^{\circ} \mathrm{C}\right)$ e de fusão $\left(\mathrm{Tm} /{ }^{\circ} \mathrm{C}\right)$ obtidos através de DMA para blendas de (PLGA/PLLA) antes degradação.

\begin{tabular}{cccc}
\hline Tipos de blendas & $\mathbf{T g}\left(\mathbf{E}^{\prime \prime}\right)$ & $\mathbf{T g}(\tan \delta)$ & $\mathbf{T g}(\tan \delta)$ \\
\hline $70: 30$ & 68 & 72 & 136 \\
$50: 50$ & 68 & 83 & 157 \\
$30: 70$ & $65 ; 81$ & 86 & 166 \\
$0: 100$ & 80 & 84 & 170 \\
\hline
\end{tabular}

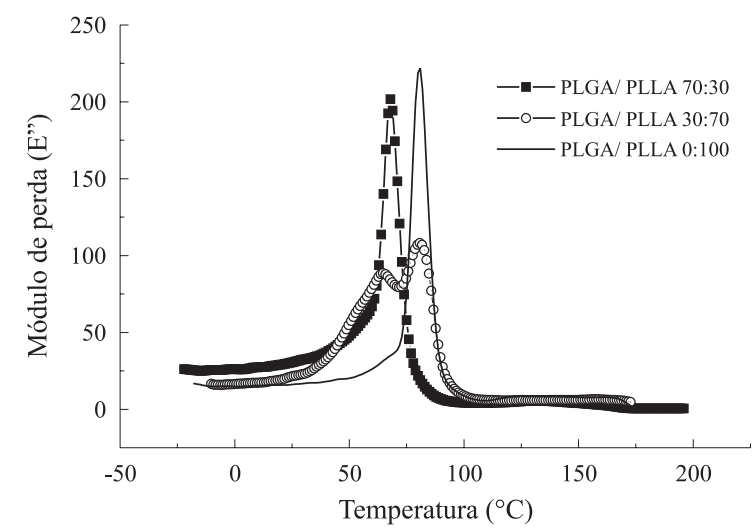

Figura 4. Curvas E" obtidas a partir de DMA para as blendas de PLGA/ PLLA nas composições 0:100, 70:30 e 30:70 antes da degradação.

PLGA está em torno de $65^{\circ} \mathrm{C}$. A temperatura de fusão do PLLA puro está em torno de $170{ }^{\circ} \mathrm{C}$ e diminui com o aumento da proporção de PLGA na blenda, o que indica que, de alguma forma, o PLGA influencia a cristalização de PLLA.

$\mathrm{Na}$ Figura 4, encontra-se o gráfico de E" obtido a partir da análise de DMA para as amostras das blendas de PLGA/PLLA nas composições 0:100, 70:30 e 30:70.

Os resultados da difratometria de raios-X mostraram que a intensidade dos picos tendeu a aumentar nas blendas à medida que a degradação ocorreu, $\mathrm{o}$ que pode ser visualizado nas Figuras 5 e 6 (respectivamente para a blenda de composição 70:30 e para a 50:50) e confirmado pelo que já foi constatado anteriormente na análise de DSC a respeito do grau de cristalinidade das amostras. Para as blendas antes da degradação, foi possível observar que a intensidade dos picos aumentou à medida que aumentou a proporção de PLLA na amostra, comparando-se os gráficos das Figuras 5 (a) e 6 (a). Este é um resultado previsível já que só o PLLA é um polímero semi- cristalino. O pico referente à presença de PLLA encontra-se em um ângulo de $17^{\circ}$. Esse comportamento repete-se também após o processo de degradação.

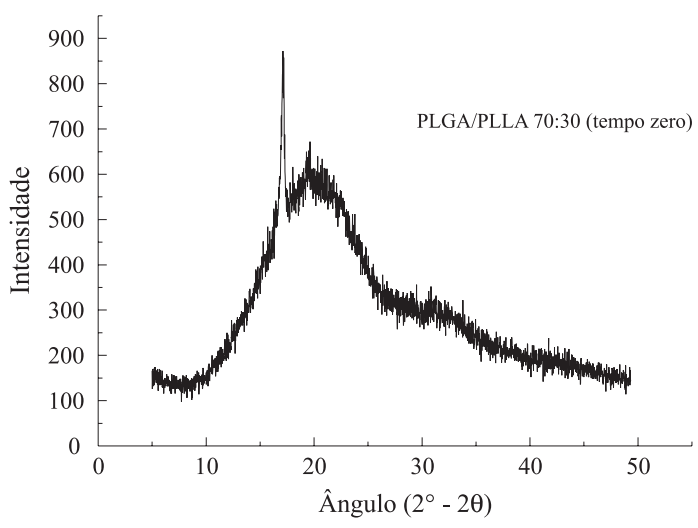

(a)

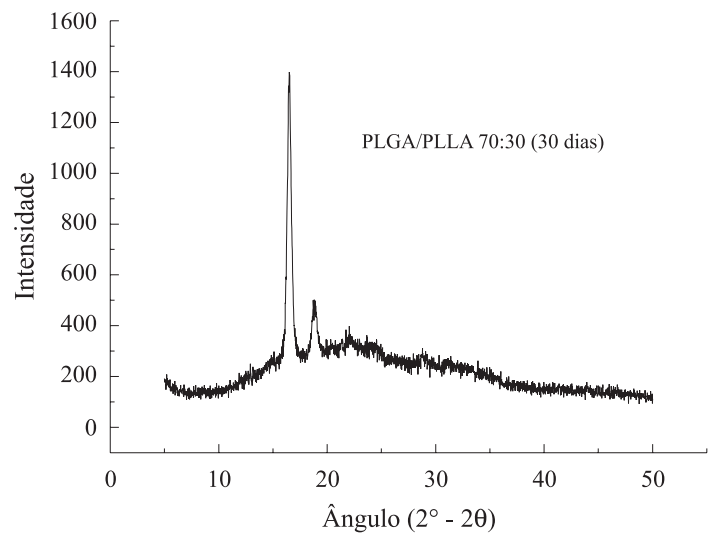

(b)

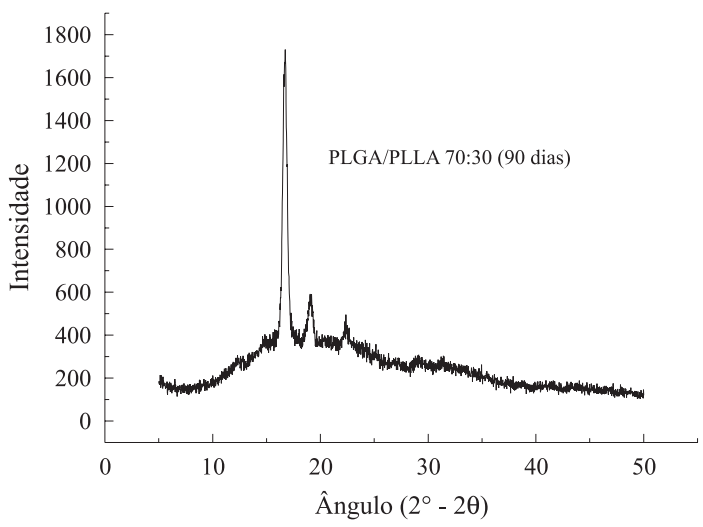

(c)

Figura 5. Curvas obtidas a partir de raios-X para a blenda PLGA/PLLA 70:30 em diferentes tempos de degradação: (a) tempo zero (b) 30 dias (c) 90 dias.

Analisando-se as micrografias das blendas de (PLGA/PLLA) obtidas a partir de MEV em vários tempos de degradação, foi possível verificar que ocorrem mudanças morfológicas em cada uma delas particulares para cada concentração. A blenda de copolímero puro apresentava uma morfologia densa antes da degradação; após 30 dias, a placa sofreu um encolhimento e 
depois deste período não foi mais possível realizar análises porque o copolímero degradou completamente. $\mathrm{Na}$ Figura 7, encontram-se as micrografias para esta blenda em tempo zero e com 30 dias de degradação.

A blenda 70:30 e a 50:50, que antes da degradação eram densas, como pode ser observado nas Figu-

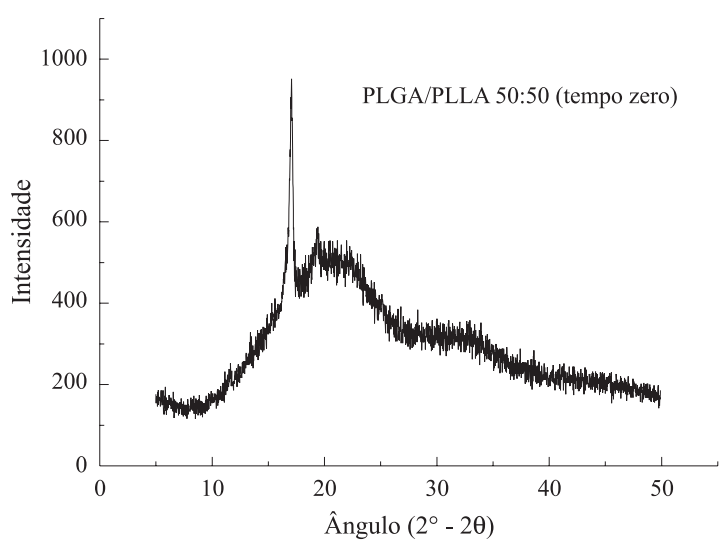

(a)

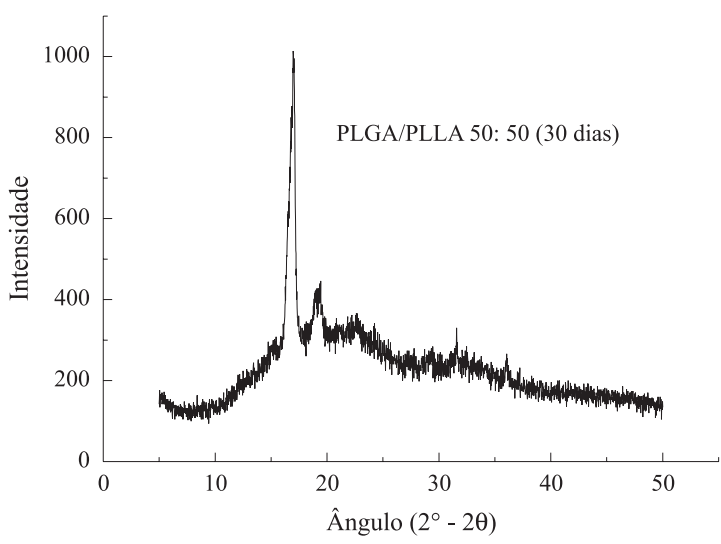

(b)

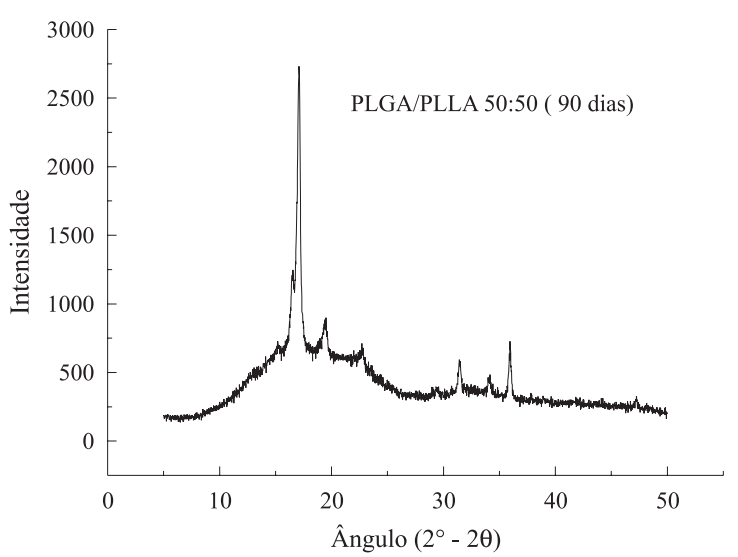

(c)

Figura 6. Curvas obtidas a partir de raios-X para a blenda PLGA/PLLA 70:30 em diferentes tempos de degradação: (a) tempo zero (b) 30 dias (c) 90 dias.

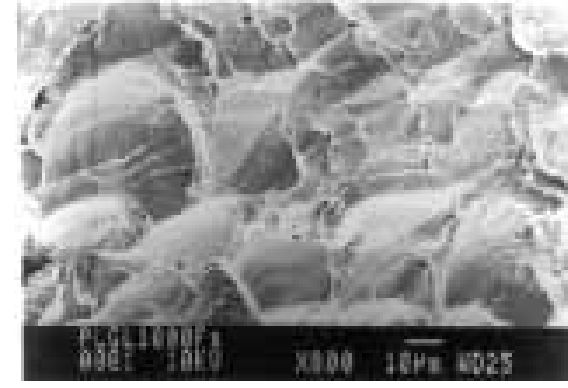

(a)

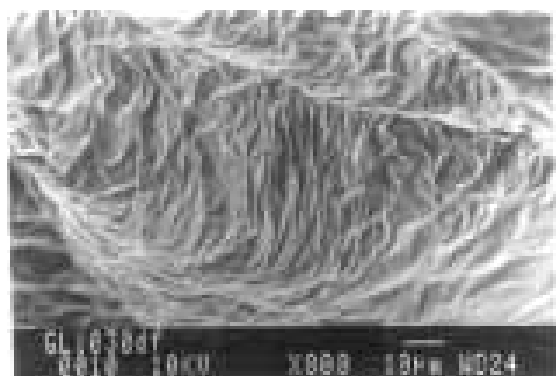

(b)

Figura 7. Micrografias obtidas para a blenda PLGA/PLLA 100:0 (a) tempo zero, (b) 30 dias.

ras 8(a) e 9 (a), respectivamente, tornam-se porosas após 30 dias, com esferas de diferentes tamanhos e fibras. As Figuras 8 (b) e 9 (b) mostram as blendas 70:30 e 50:50 com 90 e 60 dias de degradação, respectivamente, onde é possível verificar os glóbulos formados e as fibras. Com o tempo, as esferas maiores, provavelmente constituídas por PLGA, desapareceram com a degradação do mesmo. Comparando as duas blendas, pôde-se concluir que a relação entre a quantidade de glóbulos e a de fibras varia em função da proporção de PLGA na blenda: o aumento da quantidade de copolímero na blenda implica em uma morfologia globular.

A blenda 30:70 apresentou uma morfologia heterogênea antes da degradação, sugerindo a existência de duas fases: uma em alto relevo, com a presença de alguns poros, entremeada por outra lisa, em baixo relevo, como pode ser visto na Figura 10(a). Isto pode ser um indicativo de que há uma separação de fases na blenda: a fase em alto relevo constituída por PLLA e a outra por PLGA. A placa foi degradando em escamas e de uma forma mais efetiva nas bordas que no centro. Na Figura 10 (b), encontra-se uma micrografia para esta blenda com 120 dias de degradação, onde a existência de uma fase mais resistente à degradação é bastante evidente.

As micrografias obtidas para a blenda 0:100 sem degradação e com 120 dias de degradação encontram- 


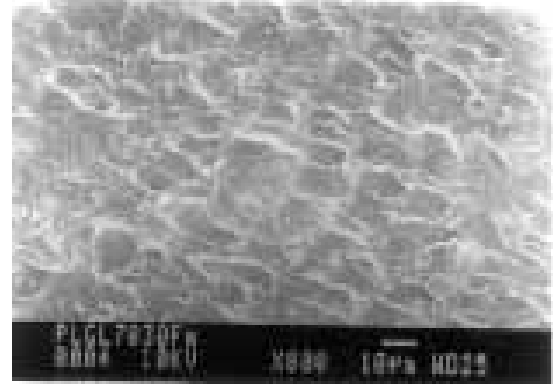

(a)

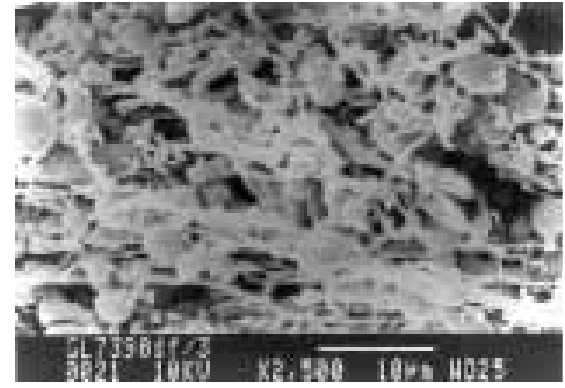

(b)

Figura 8. micrografias obtidas para a blenda PLGA/PLLA 70:30 (a) tempo zero, (b) 90 dias.

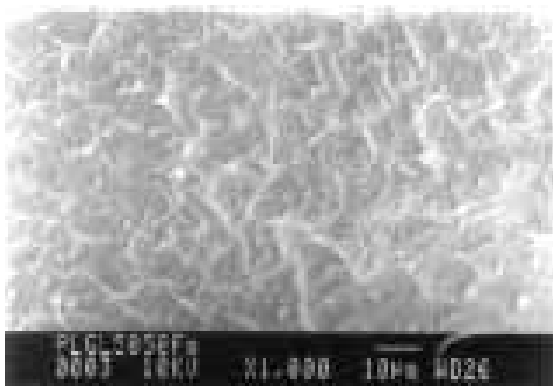

(a)

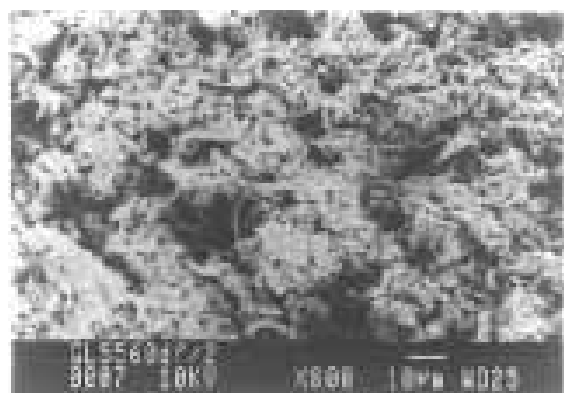

(b)

Figura 9. micrografias obtidas para a blenda PLGA/PLLA 50:50 (a) tempo zero, (b) 60 dias.

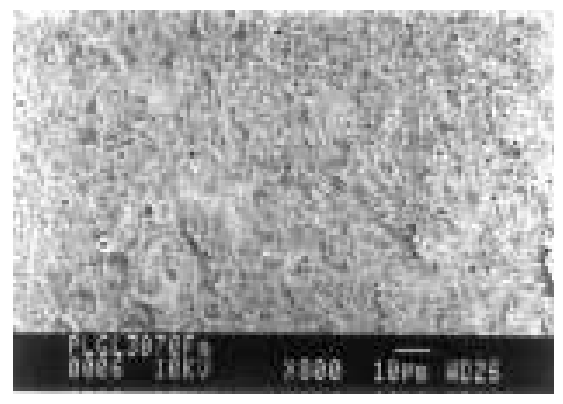

(a)

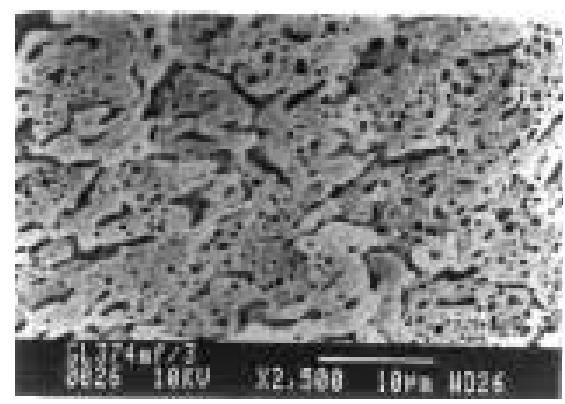

(b)

Figura 10: micrografias obtidas para a blenda PLGA/PLLA 30:70 (a) tempo zero, (b) 120 dias.

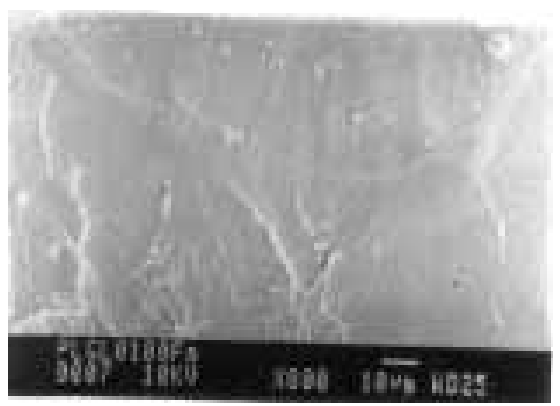

(a)

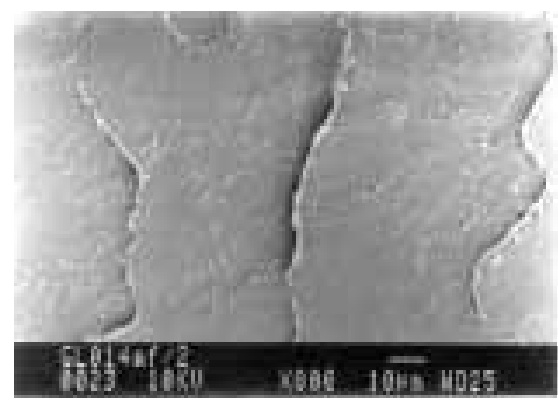

(b)

Figura 11: micrografias obtidas para a blenda PLGA/PLLA 0:100 (a) tempo zero, (b) 120 dias.

se na Figura 11 e mostram que a morfologia densa desta blenda não se alterou até 120 dias de degradação, o que concorda com os resultados obtidos nas outras análises.

\section{Conclusões}

Através da análise dos resultados apresentados anteriormente, foi possível concluir que os resulta- 
dos obtidos através da análise termogravimétrica mostraram que as blendas com os dois polímeros apresentam basicamente o mesmo comportamento quanto à sua estabilidade térmica: a degradação do PLGA levou a um aumento na $\mathrm{Td}$, que volta a diminuir lentamente ou mantém-se estável até o fim do processo de degradação. Além disso, verificou-se que os valores de Td tendem a aumentar com o aumento da concentração de PLLA na blenda e que a brusca diminuição inicial da Td para as blendas onde há copolímero, deve-se à degradação deste. Os dados de DSC mostraram que, de uma forma geral, a temperatura de cristalização (Tc) e a entalpia de cristalização $(\Delta \mathrm{Hc})$ tendem a diminuir com a degradação, ao contrário do que ocorre com o grau de cristalinidade, que aumenta com o aumento do tempo de degradação como foi observado também nas análises de raios-X. Sobre a temperatura de fusão, pode-se dizer que ela praticamente não varia em função do tempo porque seus valores dependem apenas da presença de PLLA.

A análise dos dados obtidos a partir do raios-X, mostra que a cristalinidade da amostra aumenta com o tempo de degradação, já que a intensidade dos picos aumenta com a degradação, o que pode ser confirmado pela análise de DSC. O pico referente à presença de PLLA encontra-se em um ângulo de $17^{\circ}$ e a intensidade do pico aumenta com a concentração de PLLA na blenda. A respeito dos resultados obtidos a partir de microscopia eletrônica de varredura, podese resumir que a presença de PLGA na blenda modifica drasticamente a morfologia do PLLA. Uma blenda com maior quantidade de PLGA tende a apresentar uma morfologia globular, enquanto uma menor quantidade tende a uma morfologia mais rendada.

Por fim, pode-se acrescentar, que as placas de blendas de PLGA/PLLA obtidas por fusão tem grandes chances de serem aplicadas em implantes temporários, por suas características de degradação. Um exemplo pode ser a sua aplicação em placas para neurocirurgia; a viabilidade desta aplicação ainda deve ser estudada in vivo.

\section{Agradecimentos}

Agradecemos à FAPESP pelo apoio financeiro, processo no 99/ 01381- 9 .

\section{Referências Bibliográficas}

1. Rokkanen, P.; Bostman, O.; Hirvensalo, E.; Makela, E. A.; Partio, E. K.; Patiala, H.; Vihtonen, K.; Vainionpaa, S. \& Tormala, P.- MRS Bulletin 25, p.21 (2000).

2. Schugens, C. H.; Grandfils, C.; Jerome, R.; Teyssie, P. H.; Delree, P.; Martin, D.; Malgrange, B. \& Moonen, G. - Journ. of Biomed. Mat. Res. 29, p.1349 (1995).

3. Hench, L. L. - Journ. of Biomed. Mat. Res. p.511518(1998)

4. Hench, L. L. \& Ethridge, E. C. - "Biomaterials: An Interfacial Approach”, Ed. Academic Press, New York (1982).

5. Duek, E. A. R.; Zavaglia, C. A. C. \& Belangero, W. D.Polymer 40, p.6465 (1999).

6. Younes, H. \& Cohn, D. - Europ. Polym. Journ. 24, p.765 (1998).

7. Ianasse, S.; Ambrosio, L.; Huang, S. J. \& Nicolais, L.Journ. of Appl. Polym. Scie. 54, p.1525 (1994).

8. Ferreira, B. M. P.; Zavaglia C. A. C. \& Duek, E. A. R.Mat. Res.4, p. 34 (2001).

9. Li, S. M.; Garreau , H. \& Vert M. - Journ. of Mat. Sci.: Mat. in Medic. 1, p.131(1990).

10. Pistner; H.; Bendix; D. R.; Muhling, J. \& Reuther, J. F. - Biomaterials 14, p.291 (1993).

Recebido: $10 / 08 / 01$

Aprovado: 08/10/02 